\title{
2D Structural Modulation by Odd-Even Effect of Alkyl Chains Revealed by Scanning Tunneling Microscopy at Solid/Liquid Interface
}

\author{
Yoshihiro Kikkawa* \\ National Institute of Advanced Industrial Science and Technology (AIST), Tsukuba Central 4, 1-1-1 Higashi, Tsukuba, Ibaraki \\ 305-8562, JAPAN \\ * Corresponding author: Fax: 81-29-861-3029, or e-mail: y.kikkawa@aist.go.jp
}

This review paper presents our recent progress on the formation of self-assembled two-dimensional (2D) structures, and their modulation by the odd-even effect of alkyl chains on porphyrin derivatives, isobutenyl compounds and bipyridine derivatives. The 2D structures were directly visualized by scanning tunneling microscopy (STM) at the highly oriented pyrolytic graphite (HOPG)/solvent interface. The alkyl tails in the compounds work as moleculemolecule and molecule-substrate interaction sites, and the nature of the 2D structures depended on the alkyl chain length. These STM observations revealed that, for each compound, an odd-even effect exists for a specific range of alkyl chain lengths.

Key words: odd-even effect, two-dimensional structure, solid/liquid interface, scanning tunneling microscopy

\section{INTRODUCTION}

Physical and chemical properties are sometimes greatly influenced by even only one methylene $\left(\mathrm{CH}_{2}\right)$ group difference in the alkyl chains, and alternatively changed on the basis of odd-even nature of molecular chains. This phenomenon, called the odd-even effect, has been discussed in terms of the steric repulsion of terminal methyl groups.[1] There are many examples showing the odd-even effect, such as melting point, crystalline packing, chirality, optical property, and so on.[2-7] The melting point and the enthalpy of fusion of linear alkanes changed periodically, and these alternating changes were more remarkable for alkane- $\alpha, \omega$ diols. The alkanediols with an odd number of carbon atoms are monoclinic crystals with the space group of $\mathrm{P} 2 / c$ or $\mathrm{P} 2{ }_{1} / n$, and displayed a herringbone structure. In contrast, the crystals of alkanediols with even carbon numbers were composed of parallel layers, and had an orthorhombic unit cell with the space group of $\mathrm{P} 2_{1} 2_{1} 2_{1} \cdot[8,9]$ Thus, crystalline packing structures strongly affect the melting behavior of alkanediols.

The odd-even effect is found not only for the 3D crystals, but also for 2D structures spontaneously assembled on a solid substrate. In self-assembled monolayers (SAM) can be either chemisorbed and physisorbed. The typical example of the chemisorbed SAM is alkanethiols on an $\mathrm{Au}(111)$ substrate.[10] Since the alkanethiol molecules stand with a tilt angle $\sim 30^{\circ}$ from the normal of the substrate, the number of carbon atoms in the all-trans alkanethiol determines the orientation of terminal methyl group. The odd-even effect of alkanethiol changes the SAM's surface properties, such as wettability, adhesion, friction, and so on.[11-13] In the case of physisorbed monolayers at the solid/liquid interface, molecules are almost parallel to the substrate.[14-19] The saturated fatty acids with odd numbers of carbons displayed enantiomomeric structures, whereas those with even ones formed achiral racemic domains.[20] Such structural modulations due to the odd-even effect of alkyl chains have also been reported in an anthracene derivative, dicarbamate compounds, and so on.[1,21-25]
For the study of physisorbed monolayers, the typical substrate is highly oriented pyrolytic graphite (HOPG). Scanning tunneling microscopy (STM) enables the 2D structural analysis at the molecular level. The lattice constants of the HOPG $(0.246 \mathrm{~nm})$ can be used as an internal standard, which always exists during the imaging, and thereby the correction of the lateral dimensions in the physisorbed organic monolayer is possible. The compounds to be observed are dissolved in the solvent which should be non-conductive and have low-volatility, for example, 1phenyloctane, 1,2,4-trichlorobenzene, octanol, octadecane, etc. The monolayer can be formed by simply dropping the solution on the freshly cleaved HOPG. Then, the mechanically cut STM tip is immersed in the deposited solution on the HOPG to record simultaneously the HOPG lattice as well as 2D structures of the compounds by adjusting the sample bias and tunneling current (Fig. 1). The notable advantages of using STM at the solid/liquid interface are: (1) the relationship between the molecular structure with supramolecular interaction sites and the resulting 2D ordering can be resolved in the molecular level,[14-19] (2) postreaction, such as metallation, blending, UV irradiation, etc., is possible on the surface,[26-29] and thereby (3) dynamic 2D structural changes can be followed in situ on HOPG.[3032]

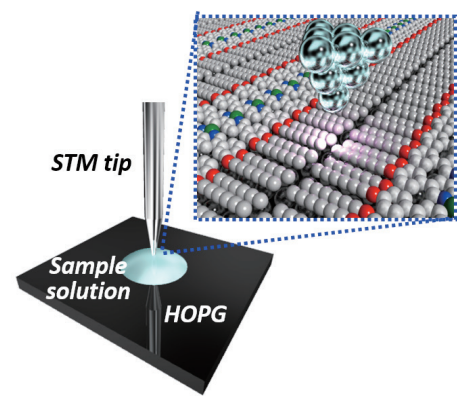

Fig. 1. Schematic drawing of the experimental set up for the STM at a solid/liquid interface. 

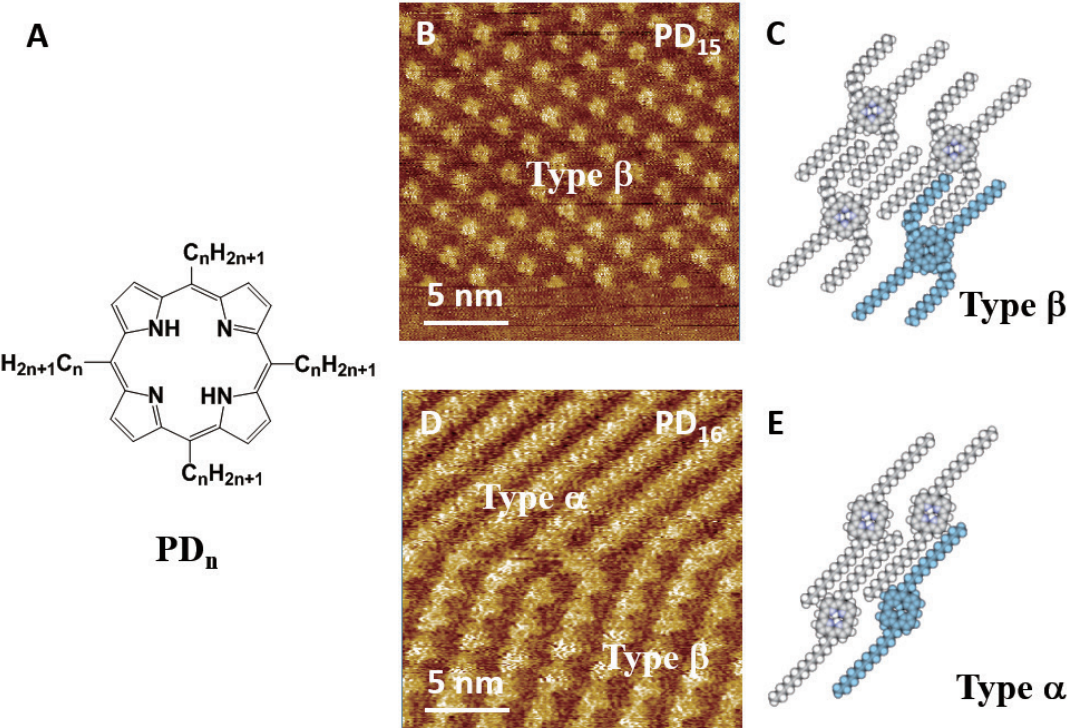

$\mathbf{F}$

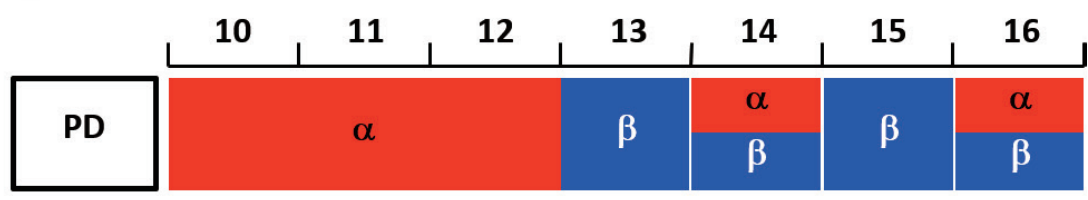

Fig. 2. Chemical structure (A) and STM images of $\mathbf{P D}_{\mathbf{n}}$ at the 1-phenyloctane/HOPG interface (B and D). The molecular models of $\alpha$ and $\beta$ structures are depicted in (E) and (C), respectively, and one molecule is colored blue. Panel (F) indicates the classification of $\alpha$ and $\beta$ structures on the basis of the STM observations. The tunneling conditions: (B) PD ${ }_{15}, I=1.5 \mathrm{pA}$, $V=-800 \mathrm{mV}$, (D) $\mathrm{PD}_{16}, I=1.5 \mathrm{pA}, V=-500 \mathrm{mV}$.

In this review, we summarize our recent progress on the formation of 2D structures, and particularly discuss the oddeven effect of alkyl tails introduced on porphyrin derivatives, isobutenyl compounds and bipyridine derivatives. The 2D structures were visualized by STM at the solid/liquid interface to reveal the molecular arrangements of each building block.

\section{PORPHYRIN DERIVATIVES [33]}

The porphyrin derivatives $\left(\mathbf{P D}_{\mathbf{n}}\right.$; the number $(\mathrm{n})$ of carbons in the alkyl chain unit is $\mathrm{n}=10-16$; Fig. 2A), namely mesotetraalkylporphyrins, were obtained in high yields, and the STM visualization was performed at the 1phenyloctane/HOPG interface. In the STM images in Fig. 2, the bright squares correspond to the $\pi$-electron rich porphyrin core, whereas the alkyl chain units are in the dark areas. Such contrasts are derived from the higher efficiency of tunneling current in $\pi$-conjugated parts compared to that in the electronically inert alkyl tails. There were two types of 2D structures that could be discriminated by the ratio of lattice constants along the $a$ - and $b$-axes $\left(R_{a b}=a / b\right)$. In the $\alpha$-type structure, half of the alkyl chains were dangling into the solvent phase, and the $R_{a b}$ value was ca. $0.6-0.7$. In contrast, all of the alkyl tails in the $\beta$ structure were attached on the HOPG surface and the $R_{a b}$ was ca. 1.0. The $R_{a b}$ value indicates that the porphyrin cores of $\alpha$-type structure are more closely-packed than those of type $\beta$. These two structures can be distinguished by the balance between the adsorption energy of alkyl chains (type $\alpha<$ type $\beta$ ) and packing density per area (type $\alpha>$ type $\beta$ ).

Figs. 2B and 2D show the STM images of $\mathbf{P D}_{15}$ and $\mathbf{P D}_{16}$, and plausible 2D molecular models for $\alpha$ and $\beta$ structures are shown in Figs. 2C and 2D, respectively. The STM observations revealed that the $\mathbf{P D}_{\mathbf{n}}(\mathrm{n}=10-12)$ formed only $\alpha$ structures, whereas the $\beta$ structure was found in the $\mathbf{P D}_{\mathbf{n}}(\mathrm{n}$ $=13-16)$. In particular, $\mathbf{P D}_{\mathbf{1 4}}$ and $\mathbf{P D}_{\mathbf{1 6}}$ displayed the phaseseparated morphologies containing both $\alpha$ and $\beta$ structures (Ex. Fig. 2D). The classifications of the 2D structures in $\mathbf{P D}_{\mathbf{n}}$ are summarized in Fig. 2F. In the $\mathbf{P D}_{\mathbf{n}}(\mathrm{n}=13-16)$, the types of $2 \mathrm{D}$ structures observed alternated, whereas $\mathbf{P D}_{\mathbf{n}}(\mathrm{n}=10$ 12) formed only $\alpha$ structures, indicating that the $2 D$ structures of $\mathbf{P D}_{\mathbf{n}}(\mathrm{n}=13-16)$ are governed by the odd-even effect of alkyl chains. Based on STM images, this effect was not observed for $\mathbf{P D}_{\mathbf{n}}(\mathrm{n} \leq 12)$. The tendency of odd-even effect remained unmodified even if the solvent was changed from 1-phenyloctane (C8) to 1-phenylnonane (C9) for the STM observations. This result suggests that the number of carbon atoms of the solvent molecule has little influence on the odd-even effect on the 2D structures of $\mathbf{P D}_{\mathbf{n}}$.

\section{ISOBUTENYL COMPOUNDS [34-36]}

Much attention has been paid for stimuli-responsive materials because of the post-tunable morphologies, properties and functions.[37,38] Among the numerous stimuli-responsive materials, we focused on the isobutenyl ether compounds, whose chemical structure can be transformed by the thermal reaction of the tandem Claisen rearrangement (TCR; also called as Hiratani-double-Claisen 

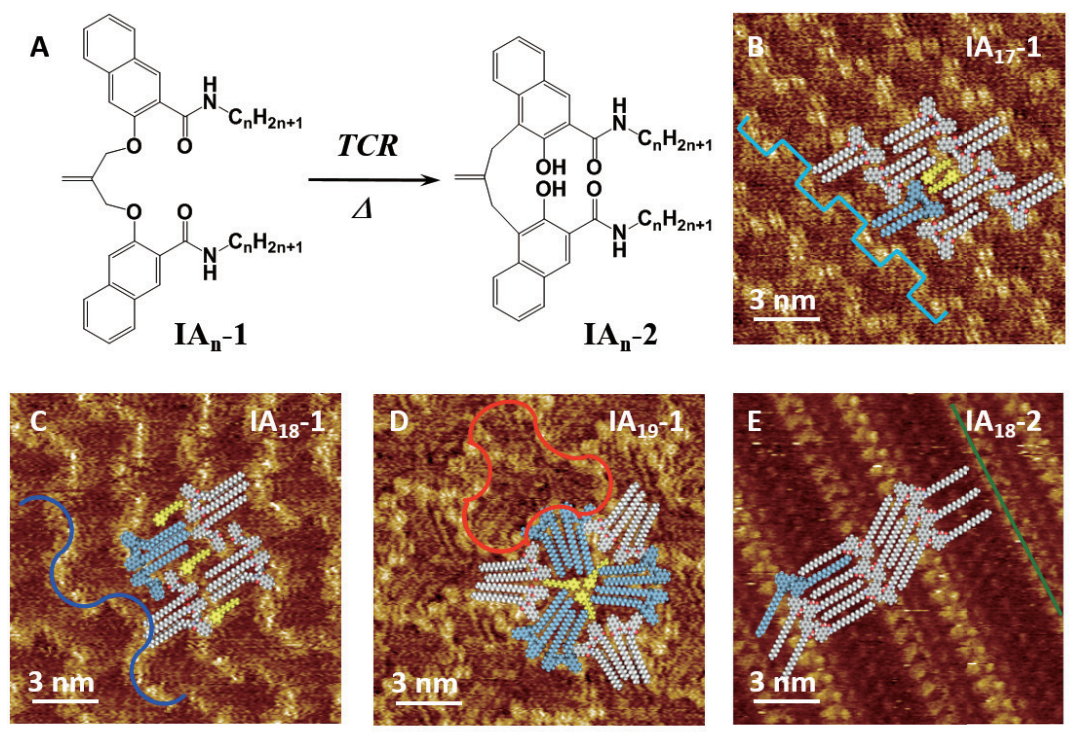

$\mathbf{F}$

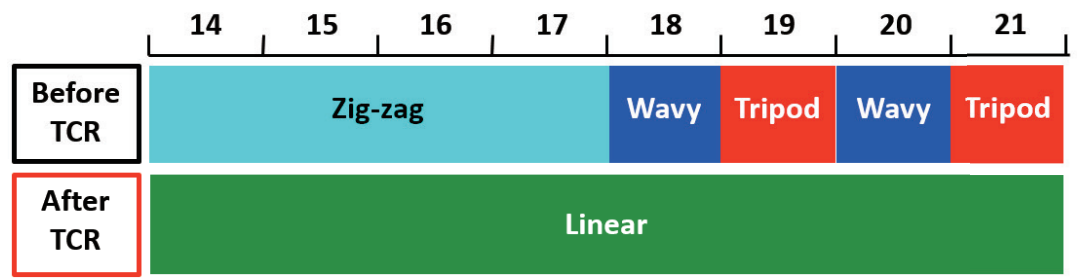

Fig. 3. Chemical structures of $\mathbf{I} \mathbf{A}_{\mathbf{n}} \mathbf{- 1}$ and $\mathbf{I} \mathbf{A}_{\mathbf{n}} \mathbf{- 2}$ (A), and typical STM images of $\mathbf{I} \mathbf{A}_{\mathbf{n}} \mathbf{- 1}$ (B-D) and $\mathbf{I} \mathbf{A}_{\mathbf{n}} \mathbf{- 2}(\mathrm{E})$ at the 1phenyloctane/HOPG interface. The molecular models are superimposed on each STM image. One of the building blocks and co-adsorbed 1-phenyl octane are colored blue and yellow, respectively. The 2D structural features are also indicated in each STM image (B: zig-zag; C: wavy; D: tripod; E: linear structures). Panel (F) indicates the classification of the 2D structures in $\mathbf{I} \mathbf{A}_{\mathbf{n}} \mathbf{- 1}$ and $\mathbf{I} \mathbf{A}_{\mathbf{n}} \mathbf{- 2}$ based on the STM observations. The tunneling conditions: (B) $I=2.1 \mathrm{pA}, V=-1300 \mathrm{mV}$, (C) $I=2.2 \mathrm{pA}, V=$ $-500 \mathrm{mV}$, (D) $I=2.0 \mathrm{pA}, V=-500 \mathrm{mV}$, (E) $I=1.1 \mathrm{pA}, V=-759 \mathrm{mV}$.

rearrangement).[39,40] The isobutenyl compounds bearing alkyl chains on their amide groups were prepared $\left(\mathbf{I A}_{\mathbf{n}} \mathbf{- 1}\right.$; Fig. $3 \mathrm{~A}$ ), and the effects of alkyl chain length and TCR on the 2D structures were studied to pave the way for the fabrication of thermally tunable intelligent nano-materials.

STM observations of $\mathbf{I} \mathbf{A}_{\mathbf{n}}-\mathbf{1}$ were performed at the HOPG/1phenyloctane interface. The naphthalene and alkyl chain units were visualized as the bright and dark contrasts, respectively. Basically, the hydrogen bonds between the isobutenyl ether oxygen atom and the amide $\mathrm{NH}$ proton oriented the alkyl chains in the same direction. The $\mathbf{I} \mathbf{A}_{\mathbf{n}} \mathbf{- 1}(\mathrm{n}=14-17)$ formed the zig-zag structure (Fig. 3B), in which the direction of alkyl chains are alternately changed. For $\mathbf{I A}_{\mathbf{1 8}} \mathbf{- 1}$, a wavy structure was formed (Fig. 3C), whereas $\mathbf{I} \mathbf{A}_{\mathbf{1 9}} \mathbf{- 1}$ and $\mathbf{I A}_{\mathbf{2 1}} \mathbf{- 1}$ displayed the $\mathrm{C} 3$ symmetric tripod structure (Fig. 3D). In the wavy structure, molecules formed as pairs, and one of the alkyl chains dangled into the solvent phase. In contrast, all the alkyl chains in the tripod structure lied on the HOPG surface. Exceptionally, both wavy and tripod structures were found as biphasic separation in the $\mathbf{I A}_{\mathbf{2 0}} \mathbf{- 1}$. However, changing the solvent from 1-phenyloctane to 1-phenylnonane allowed $\mathbf{I A}_{\mathbf{2 0}} \mathbf{- 1}$ to form only the wavy structure.

After the TCR, the ether functions of $\mathbf{I} \mathbf{A}_{\mathbf{n}} \mathbf{- 1}$ were converted to hydroxyl groups and a new $\mathrm{C}-\mathrm{C}$ bond was formed in $\mathbf{I} \mathbf{A}_{\mathbf{n}}{ }^{-}$ 2. Although TCR disrupted the hydrogen bonds between the isobutenyl ether oxygen atom and the amide NH proton, new intramolecular hydrogen bonds formed between the hydroxyl and carbonyl groups. The TCR reaction could be performed both in solution and at the HOPG/solvent interface. All the 2D structures of $\mathbf{I} \mathbf{A}_{\mathbf{n}} \mathbf{- 2}(\mathrm{n}=14-21)$ converged into the same close-packed linear structure (Fig. 3E), in which every other naphthalene and its interdigitated alkyl chains are flipped in opposite directions. A plausible mechanism for the 2D structural transformation was the shortening of the intramolecular distance between the naphthalene units for $\mathbf{I} \mathbf{A}_{\mathbf{n}} \mathbf{- 2}$. Intramolecular hydrogen bonds exist in both $\mathbf{I A} \mathbf{A}_{\mathbf{n}} \mathbf{- 1}$ and $\mathbf{I} \mathbf{A}_{\mathbf{n}} \mathbf{- 2}$, however the position of these bonds is different. After TCR, the distance between the naphthalene units of $\mathbf{I} \mathbf{A}_{\mathbf{n}} \mathbf{- 2}$ became shorter than in $\mathbf{I} \mathbf{A}_{\mathbf{n}}-\mathbf{1}$, and therefore every other naphthalene unit of $\mathbf{I} \mathbf{A}_{\mathbf{n}} \mathbf{- 2}$ should be oriented in the opposite direction to reduce the steric hindrance of the hydroxyl groups formed after the TCR.

In summary, the 2D structures of $\mathbf{I} \mathbf{A}_{\mathbf{n}} \mathbf{- 1}(\mathrm{n} \geq 18)$ displayed either wavy or tripod structures due to an odd-even effect of alkyl chain units (Fig. 3F). In contrast, the $\mathbf{I A}_{\mathbf{n}} \mathbf{- 1}(\mathrm{n} \leq 17)$ showed the same 2D structures with zig-zag orientation of isobutenyl compounds, regardless of the alkyl chain length. However, after the TCR, all the 2D structures of $\mathbf{I} \mathbf{A}_{\mathbf{n}} \mathbf{- 2}(\mathrm{n}=$ $14-21$ ) converged into the same linear structures, and the odd-even effect was completely cancelled. 
A
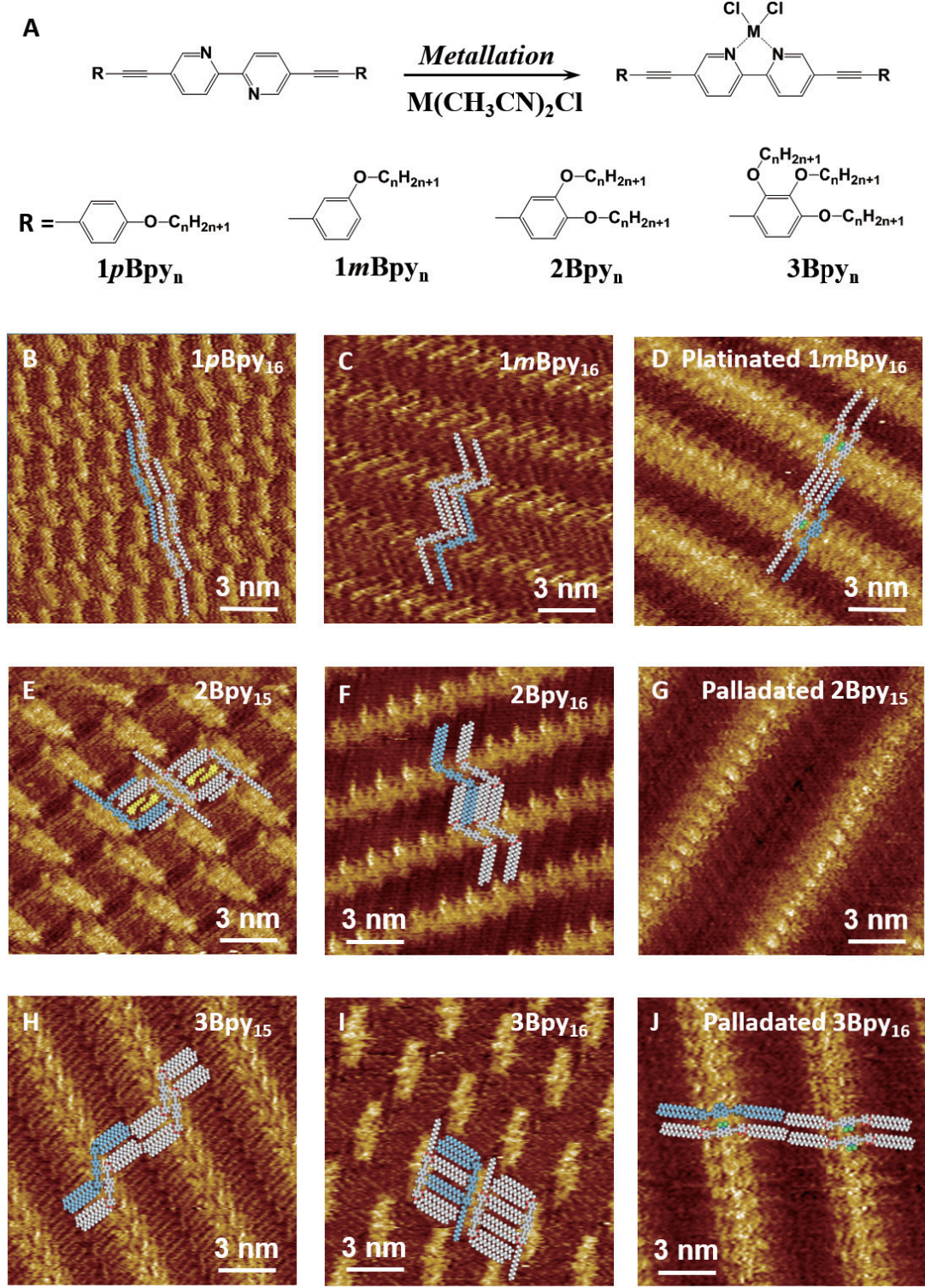

K

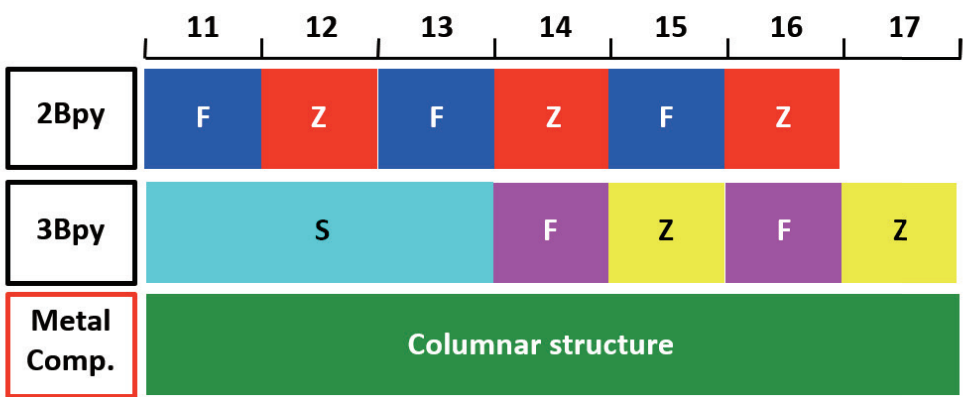

Fig. 4. Chemical structures of $\mathbf{B p y}_{\mathbf{n}} \mathbf{s}$ (A). Typical STM images of $\mathbf{1 p B p y}_{16}$ (B), $\mathbf{1} \mathbf{m} \mathbf{B p y}_{16}$ (C), platinated $\mathbf{1} m \mathbf{B p y} \mathbf{y}_{16}$ (D), $\mathbf{2} \mathbf{B p y}_{15}$ (E), 2Bpy $\mathbf{B}_{16}(\mathrm{~F})$, palladated $\mathbf{2} \mathbf{B p y}_{15}(\mathrm{G}), \mathbf{3} \mathbf{B p y} \mathbf{y}_{15}(\mathrm{H}) \mathbf{3} \mathbf{B p y}_{16}(\mathrm{I})$, palladated $\mathbf{3} \mathbf{B p y}_{16}(\mathrm{~J})$ at the 1-phenyloctane/HOPG interface. The molecular models are superimposed on each STM image, and one of the building blocks and co-adsorbed 1phenyl octane are colored blue and yellow, respectively. In panel $(\mathrm{K})$, the 2D structures of $\mathbf{B p y} \mathbf{n} \mathbf{s}$ before and after the metal coordination were classified from the viewpoint of the shape of molecular building blocks. Tunneling conditions: (B) $I=75$ $\mathrm{pA}, V=-1000 \mathrm{mV}$,(D) $I=70 \mathrm{pA}, V=-1000 \mathrm{mV}$,(D) $I=1.7 \mathrm{pA}, V=-622 \mathrm{mV}$, (E) $I=1.8 \mathrm{pA}, V=-292 \mathrm{mV}$, (F) $I=1.2 \mathrm{pA}, V$ $=-288 \mathrm{mV},(\mathrm{G}) I=1.2 \mathrm{pA}, V=-273 \mathrm{mV}$, (H) $I=1.1 \mathrm{pA}, V=-660 \mathrm{mV}$, (I) $I=1.6 \mathrm{pA}, V=-650 \mathrm{mV}$, (J) $I=1.3 \mathrm{pA}, V=-654$ $\mathrm{mV}$. 


\section{BIPYRIDINE DERIVATIVES [41-44]}

In addition to studying the effect of the alkyl chain length, the influence of the number of alkyl chains was also studied for bipyridine derivatives $\left(\mathbf{B p y} \mathbf{y}_{\mathbf{n}}\right)$. In this context, $\mathbf{B p y} \mathbf{y}_{\mathbf{n}} \mathbf{s}$ substituted by different numbers and lengths of alkyl chains $(\mathrm{n}=11-17)$ were designed and synthesized, as shown in Fig. 4A. The Bpys are good ligands for metals, therefore the 2D structural changes occurring upon metal complexation were also studied at the HOPG/1-phenyloctane interface.

The $\mathbf{B p y}_{\mathbf{n}} \mathbf{s}$ substituted with a single alkyl chain at both $p$ positions $\left(\mathbf{1} p \mathbf{B p y} \mathbf{y}_{\mathbf{n}}\right)$ were almost linearly aligned (Fig. 4B), and those substituted at both $m$-positions $\left(\mathbf{1} \mathbf{m} \mathbf{B p y} \mathbf{y}_{\mathbf{n}}\right)$ showed Z-shaped morphology with interdigitated alkyl chains (Fig. 4C), regardless of the alkyl chain length. After palladation, a 2D structural change occurred for $1 p \mathbf{B p y}$, and an interdigitation of the alkyl chains was introduced (see ref.[43]). In the case of $\mathbf{1} \boldsymbol{m} \mathbf{B p y} \mathbf{y}_{\mathbf{n}}$, the same 2D structures were observed at the solid/liquid interface before and after the palladation, nevertheless the palladation was confirmed in solution. This result suggests that the 2D structure of $\mathbf{1} \mathbf{m} \mathbf{B p y _ { n }}$ is too stable to change its molecular orientation even after palladation (see ref.[43]), and that the decoordinated $\mathbf{1} \mathbf{m} \mathbf{B p y _ { \mathbf { n } }}$ from the metal enabled the formation of the close-packed 2D structure because of the labile nature of the Pd complex. [45] However, the platination of $\mathbf{1} \mathbf{m} \mathbf{B p y}_{\mathbf{n}}$ allowed structural changes, as shown in Fig. 4D. Both metallated $\mathbf{1 B p y}_{\mathbf{n}} \mathbf{s}$ showed similar molecular arrangements, and it is suggested that no odd-even effect was observed for both $p$ - and $m$ substituted $\mathbf{1} \mathbf{B p y}_{\mathbf{n}} \mathbf{s}$ before and after the metallation.

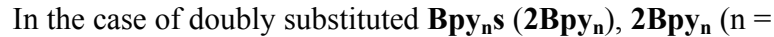
odd: $11,13,15)$ adopted an $F$-shaped conformation, and the vacant space was filled with 1-phenyloctane solvent molecules (Fig. 4E). In contrast, Z-shaped molecules of $\mathbf{2 B p y}_{\mathbf{n}}(\mathrm{n}=$ even: $12,14,16)$ had interdigitated alkyl chain units (Fig. 4F), suggesting that the 2D structures of $\mathbf{2} \mathbf{B p} \mathbf{y}_{\mathbf{n}}$ were formed on the basis of the odd-even effect of alkyl chains.

The 2D structures of $\mathbf{B p y}_{\mathbf{n}} \mathbf{s}\left(\mathbf{3} \mathbf{B p y} \mathbf{y}_{\mathbf{n}}\right)$ substituted with three alkyl chains were categorized into two groups: the $\mathbf{3} \mathbf{B p y}_{\mathbf{n}}(\mathbf{n}$ $=11-13)$ were straightly aligned and flatly attached on the HOPG surface; the $\mathbf{3} \mathbf{B p y} \mathbf{y}_{\mathbf{n}}(\mathrm{n}=14-17)$ showed the odd-even effect for alkyl chain length. An $F$ conformation was observed for $\mathbf{3} \mathbf{B p y}_{14}$ and $\mathbf{3} \mathbf{B p y} \mathbf{y}_{\mathbf{1 6}}$ (Fig. 4I), whereas a $\mathrm{Z}$ form with interdigitated alkyl chains was seen for $\mathbf{3} \mathbf{B p y}_{\mathbf{1 5}}$ and $\mathbf{3 B p y}_{17}$ (Fig. 4H). Therefore, an odd-even effect emerged for $\mathbf{3 B p y} \mathbf{y}_{\mathbf{n}}$ within only a limited range of alkyl chain lengths.

The palladation of $\mathbf{2} \mathbf{B p y} \mathbf{y}_{\mathbf{n}}$ and $\mathbf{3} \mathbf{B} \mathbf{p y} \mathbf{y}_{\mathbf{n}}$ was confirmed by (1) the characteristic color change of the solution containing both the ligand and $\mathrm{Pd}\left(\mathrm{CH}_{3} \mathrm{CN}\right)_{2} \mathrm{Cl}_{2}$ and (2) the downfield shift of the $\alpha$ proton of bipyridine in the ${ }^{1} \mathrm{H}$ NMR compared to the same proton in the free ligand. For the STM observations, both in situ and ex situ metallation were separately carried out. Namely, the $\mathrm{Pd}\left(\mathrm{CH}_{3} \mathrm{CN}\right)_{2} \mathrm{Cl}_{2}$ solution was added to the already-existing drop of the $\mathbf{B p y}_{\mathbf{n}} \mathbf{s}$ on the HOPG for the in situ experiment, whereas the ex situ experiment was performed by pre-mixing the $\mathbf{B p y}_{\mathbf{n}} \mathbf{s}$ and $\mathbf{P d}\left(\mathrm{CH}_{3} \mathrm{CN}\right)_{2} \mathrm{Cl}_{2}$ before the STM studies. In both cases, the molecules of $\mathbf{2 B p y}$ and $\mathbf{3 B p y} \mathbf{n}_{\mathbf{n}}$ assembled as columnar structures without interdigitation of alkyl chains (Figs. 4G and 4J). Especially in the case of $\mathbf{3} \mathbf{B p y}_{\mathbf{n}}$, one of the alkyl chains, possibly in the $o$ position, is not attached to the HOPG surface, resulting in the same 2D structures as $\mathbf{2} \mathbf{B p y} \mathbf{y}_{\mathbf{n}}$. Such structural changes could be explained in terms of a variation of molecular width upon metal coordination. After the palladation, the molecular width at the bipyridine core $(0.75 \mathrm{~nm})$ changed to $0.91 \mathrm{~nm}$, which is almost identical to the width of two peripheral alkyl chains $(0.46 \times 2=0.92 \mathrm{~nm})$. Thus, the straight conformation of the palladated $\mathbf{2} \mathbf{B p y} \mathbf{y}_{\mathbf{n}}$ and $\mathbf{3} \mathbf{B p y} \mathbf{y}_{\mathbf{n}}$ is favorable for the densely packed $2 \mathrm{D}$ structures.

The 2D structures of $\mathbf{2} \mathbf{B p y} \mathbf{y}_{\mathbf{n}}$ and $\mathbf{3} \mathbf{B p y} \mathbf{y}_{\mathbf{n}}$ before and after the metallation are summarized in Fig. 4K. The 2D structures of $\mathbf{2} \mathbf{B p y}_{\mathbf{n}}(\mathrm{n}=11-16)$ and $\mathbf{3} \mathbf{B p y} \mathbf{n}(\mathrm{n}=14-17)$ were dominated by the odd-even effect of alkyl chains, but this was not the case for $\mathbf{3} \mathbf{B p y}_{\mathbf{n}}(\mathrm{n}=11-13)$. Therefore, not only the alkyl chain length but also the number of alkyl chains influences the emergence of the odd-even effect. However, after metallation, the odd-even effect was quenched possibly due to the change in the intermolecular interaction between the bipyridine core and alkyl chain units.

\section{CONCLUSIONS AND OUTLOOK}

We have summed up our recent work on the $2 \mathrm{D}$ structure formations that were governed by the odd-even effect of alkyl chains. For the studied compounds of $\mathbf{P D}_{\mathbf{n}} \mathbf{s}, \mathbf{I} \mathbf{A}_{\mathbf{n}} \mathbf{s}$ and $\mathbf{B p y}_{\mathbf{n}} \mathbf{s}$, a specific alkyl chain length range showed the oddeven effect. In the cases of $\mathbf{I A}_{\mathbf{n}} \mathbf{s}$ and $\mathbf{B p y} \mathbf{y}_{\mathbf{n}} \mathbf{s}$, TCR and metallation both cancelled the odd-even effect, resulting in the structural conversion possibly because of the change in the intermolecular interaction as well as conformational limitation of the components.

We will continue our efforts to produce well-ordered patterns with a single-nanoscale pitch, which cannot be achieved by the conventional top-down strategy. Indeed, the general technique to create various $2 \mathrm{D}$ structures is to introduce the directional interaction sites into the molecular building blocks; nevertheless, the odd-even effect is also helpful for the fabrication of complicated and interesting 2D nano-architectures. A deeper understanding of intermolecular interactions in supramolecular 2D structures will provide the effective integration of surface-assisted $3 \mathrm{D}$ structures and lead to the practical application of nanomaterials. In addition, multi-component and post-tunable materials composed of stimuli-responsive building blocks will be one of the most fascinating candidates to further develop the bottom-up fabrication system of nanostructures.

\section{ACKNOWLEDGEMENTS}

This work has been partially supported by a grant from the Sumitomo Foundation, JSPS (23750137) and MEXT KAKENHI (21106522; 23106722). The Open Partnership Joint Projects of JSPS Bilateral Joint Research Projects are also acknowledged. The author is very grateful to all of the collaborators: Dr. M. Kanesato, Prof. K. Hiratani, Dr. E. Koyama, Dr. S. Tsuzuki, Dr. H. Tokuhisa, Dr. K. Miyake, Dr. K. Omori, Ms. K. Fujiwara, Ms. M. Takahashi (AIST, Japan), and Prof. T. S. Balaban (Marseille, France). In addition, the author would like to express his great thanks to Dr. Jennifer Wytko (Strasbourg, France) for the English correction of present manuscript.

\section{REFERENCES}

[1] F. Tao and S. L. Bernasek, Chem. Rev, 107, 1408-1453 (2007).

[2] A. D. Bond, New J. Chem., 28, 104-114 (2004).

[3] A. Prudencio, R. C. Schmeltzer and K. E. Uhrich, Macromolecules, 38, 6895-6901 (2005).

[4] G. Knothe and O. Dunn, J. Am. Oil Chem. Soc., 86, 843856 (2009). 
[5] X. Weng, C. Y. Li, S. Jin, D. Zhang, J. Z. Zhang, F. Bai, F. W. Harris and S. Z. D. Cheng, Macromolecules, 35, 9678$9686(2002)$

[6] S. Umadevi, A. Jáklib and B. K. Sadashiva, Soft Matter, 2, 875-885 (2006).

[7] D. A. M. Egbe, C. Ulbricht, T. Orgis, B. Carbonnier, T. Kietzke, M. Peip, M. Metzner, M. Gericke, E. Birckner, T. Pakula, D. Neher and U. -W. Grummt, Chem. Mater., 17, 6022-6032 (2005).

[8] V.R. Thalladi, R. Boese and H. C. Weiss, Angew. Chem. Int. Ed., 239, 918-922 (2000).

[9] E. Badea, B. Nowicka and G. Della Gatta, J. Chem. Thermodyn., 68, 90-97 (2014).

[10] R. K. Smith, P. A. Lewis and P. Weiss, Prog. Surf. Sci. 75, 1-68 (2004).

[11] R. Colorado, Jr. and T. R. Lee, Langmuir, 19, 3288-3296 (2003).

[12] B. Park, M. Chandross, M. J. Stevens and G. S. Grest, Langmuir, 19, 9239-9245 (2003).

[13] P. T. Mikulski, L. A. Herman and J. A. Harrison, Langmuir, 21, 12197-12206 (2005).

[14] S. De Feyter and F. C. De Schryver, J. Phys. Chem. B. 109, 4290-4302 (2005).

[15] K. E. Plass, A. L. Grzesiak and A. J. Matzger, Acc. Chem. Res. 40, 287-293 (2007).

[16] J. A. A. W. Elemans, S. Lei and De Feyter, S. Angew. Chem. Int. Ed. 48, 7298-7332 (2009).

[17] J. Otsuki, Coord. Chem. Rev. 254, 2311-2341 (2010).

[18] C. -A. Palma, A. Ciesielski, M. Bonini and P. Samorì, "Functional Supramolecular Architectures" Ed. by P. Samorì and F. Cacialli) pp., Willey-VCH, Weinheim, 423-455 (2011) [19] Y. Kikkawa, Polym. J., 45, 255-260 (2013).

[20] F. Tao and S. L. Bernasek, J. Phys. Chem. B, 109, 62336238 (2005).

[21] Y. Wei, K. Kannappan, G. W. Flynn and B. M. Zimmt, J. Am. Chem. Soc., 126, 5318-5322 (2004).

[22] K. Kim, K. E. Plass and A. J. Matzger, J. Am. Chem. Soc., 127, 4879-4887 (2005)

[23] L. Xu, X. miao, B. Zha, K. Miao and W. Deng,, J. Phys. Chem. C., 117, 12707-12714 (2013).

[24] L. Xu, X. miao, B. Zha, K. Miao and W. Deng,, Chem. Asian J., 8, 926-933 (2013).

[25] E. Ghijsens, O. Ivasenko, K. Tahara, H. Yamaga, S. Itano, T. Balandina, Y. Tobe and S. De Feyter, ACS Nano, 7, 8031-8042 (2013)

[26] Y. Okawa and M. Aono, Nature, 409, 683-684 (2001).
[27] S. De Feyter and F. C. De Schryver, Chem. Soc. Rev. 32, 139-150 (2003).

[28] L. Piot, R. M. Meudtner, T. E. Malah, S. Hecht and P. Samorì, Chem. Eur. J. 15, 4788-4792 (2009).

[29] Y. Kikkawa, H. Kihara, M. Takahashi, M. Kanesato, T. S. Balaban and J. -M. Lehn, J. Phys. Chem. B., 114, 16718 16722 (2010).

[30] S. De Feyter, A. Gesquière, M. M. Abdel-Mottaleb, P. C. M. Grim, F. C. De Schryver, C. Meiners, M. Sieffert, S. Valiyaveettil and K. Müllen, Acc. Chem. Res., 33, 520-531 (2000).

[31] S. Uemura, R. Tanoue, N. Yilmaz, A. Ohira, and M. Kunitake, Materials 3, 4252-4276 (2010).

[32] Y. Kikkawa, S. Kubota, T. Karatsu, A. Kitamura, M. Kanesato and S. Yagai, J. Nanosci. Nanotech., 10, 803-808 (2010).

[33] R. Plamont, Y. Kikkawa, M. Takahashi, M. Kanesato, M Giorgi, A. C. K. Shun, C. Roussel and T. S. Balaban, Chem. Eur. J., 19, 11293-11300 (2013).

[34] K.Omori, Y. Kikkawa, H. Tokuhisa, M. Kanesato and K. Hiratani, Colloids Surf. A, 356, 58-62 (2010).

[35] K.Omori, Y. Kikkawa, M. Kanesato and K. Hiratani, Chem. Commun., 46, 8008-8010 (2010).

[36] Y. Kikkawa, K.Omori, M. Takahashi, M. Kanesato and K. Hiratani, Org. Biomol. Chem. 10, 8087-8094 (2012).

[37] M. Motornov, Y. Roiter, I. Tokarev and S. Minko, Prog. Polym. Sci., 35, 174-211 (2010).

[38] J. Hu, G. Zhang and S. Liu, Chem. Soc. Rev., 41, 59335949 (2012)

[39] M. Albrecht, R. Osetska, R. and R. Froehlich, Synlett 6, 924-926 (2006)

[40] K. Hiratani and M. Albrecht, Chem. Soc. Rev. 37, 24132421 (2008).

[41] Y. Kikkawa, E. Koyama, S. Tsuzuki, K. Fujiwara, K. Miyake, H. Tokuhisa and M. Kanesato, Langmuir, 22, 69106914 (2006).

[42] Y. Kikkawa, E. Koyama, S. Tsuzuki, K. Fujiwara, K. Miyake, H. Tokuhisa and M. Kanesato, Chem. Commun., 1343-1345 (2007).

[43] Y. Kikkawa, E. Koyama, S. Tsuzuki, K. Fujiwara and M. Kanesato, Langmuir, 26, 3376-3381 (2010).

[44] Y. Kikkawa, E. Koyama, M. Aoyagi, N. Schneider, M. Takahashi, K. Fujiwara and M. Kanesato, Supramol. Chem., 23, 9-12 (2011).

[45] M. Fujita, F. Ibukuro, K. Yamaguchi, K. Ogura, J. Am. Chem. Soc. 117, 4175-4176 (1995).

(Received March 19, 2014; Accepted April 14, 2014) 